\title{
Correction to: EuroSurg-1 study: an opportunity to encourage student- driven surgical research in Italy
}

\author{
Italian Committee of EuroSurg Collaborative ${ }^{1}$
}

Published online: 14 March 2019

(c) Springer Nature Switzerland AG 2019

\section{Correction to: Tech Coloproctol (2016) 20:423-424 https://doi.org/10.1007/s10151-016-1427-5}

Unfortunately, the collaborators of the above mentioned article was missed out in the affiliation of the original publication. The details of them are listed below.

\section{EuroSurg Italian Committee}

Michael Bath ${ }^{10}$, Valeria Farina ${ }^{1}$, Caterina Foppa ${ }^{2}$, Laura Gavagna $^{3}$, Pietro Naccari ${ }^{4}$, Sandro Pasquali ${ }^{5}$, Francesco Pata $^{6}$, Gianluca Pellino ${ }^{7}$, Bruno Sensi ${ }^{8}$, Alessando Sgro ${ }^{9}$.

${ }^{1}$ Department of Surgical Sciences, University of Turin, Turin, Italy.

${ }^{2}$ Unit of General and Endocrine Surgery, Department of Surgery and Translational Medicine, University of Florence, Florence, Italy.

${ }^{3}$ Department of Morphology, Surgery and Experimental Medicine, University of Ferrara, Italy.

${ }^{4}$ University of Trieste, Trieste, Italy.

${ }^{5}$ Department of Surgery, Oncology and Gastroenterology, University of Padova, Padova, Italy.

${ }^{6}$ Department of Surgery, Sant'Antonio Abate Hospital, Gallarate, Italy.

${ }^{7}$ Department of Medical, Surgical, Neurological, Metabolic and Ageing Sciences, Second University of Naples, Naples, Italy.

${ }^{8}$ Department of Surgery, Tor Vergata University Hospital, Rome, Italy.

${ }^{9}$ University of Pavia, Pavia, Italy.

The original article can be found online at https://doi.org/10.1007/ s10151-016-1427-5.

Italian Committee of EuroSurg Collaborative

eurosurgitalia@gmail.com

1 Second University of Naples, Piazza Miraglia 2,

80138 Naples, Italy
${ }^{10}$ Department of Surgery, University of Cambridge, Cambridge, UK.

\section{Supporting UK Trainees}

Aneel Bhangu ${ }^{1}$, Stephen J. Chapman ${ }^{2}$, Thomas M. Drake ${ }^{3}$, J. Edward Fitzgerald ${ }^{4}$, Dmitri Nepogodiev ${ }^{1}$.

${ }^{1}$ Academic Department of Surgery, University of Birmingham, Birmingham B15 2TH, UK.

${ }^{2}$ University of Leeds, Leeds, UK.

${ }^{3}$ Department of Oncology \& Metabolism, University of Sheffield, Sheffield, UK.

${ }^{4}$ Kings College London, London, UK.

\section{Supporting Italian Consultants}

Alberto Arezzo ${ }^{1}$, Mario Morino ${ }^{1}$, Michele Rubbini ${ }^{2}$, Francesco Selvaggi ${ }^{3}$, Giuseppe Sica ${ }^{4}$.

${ }^{1}$ Department of Surgical Sciences, University of Turin, Turin, Italy.

${ }^{2}$ Department of Morphology, Surgery and Experimental Medicine, University of Ferrara, Italy.

${ }^{3}$ Department of Medical, Surgical, Neurological, Metabolic and Ageing Sciences, Second University of Naples, Naples, Italy.

${ }^{4}$ Department of Surgery, Tor Vergata University Hospital, Rome, Italy.

Publisher's Note Springer Nature remains neutral with regard to jurisdictional claims in published maps and institutional affiliations. 